\title{
Dra. Emma TORRES-ROMAY*
}

Profesora titular. Facultad de Ciencias Sociales y de la Comunicación. Universidad de Vigo. España. emmatr@uvigo.es

\section{Silvia GARCÍA-MIRÓN*}

Profesora ayudante. Facultad de Ciencias Sociales y de la Comunicación. Universidad de Vigo. España. silviamiron@uvigo.es

La evolución de la estrategia publicitaria. Del brand management a la marca adaptada y el nuevo papel del consumidor Strategist Advertising evolution. From brand management to personalized brand and consumers new role

\section{Resumen}

El presente trabajo de investigación pretende realizar un repaso por los cambios que se han podido producir en los procesos de planificación estratégica publicitaria. Para ese fin se ha realizado un seguimiento de las principales tendencias comunicativas en este ámbito, intentando identificar los modelos actuales para poder así, comparar la realidad actual con lo que sucedía antes de la crisis. El punto de partida en este caso está en el hecho de que, tal y como preveía la Nueva Teoría Estratégica, la recuperación del ser humano es la base del funcionamiento de la estrategia publicitaria actual, donde las marcas deben adaptarse a las preferencias del consumidor de una forma casi individual y los mensajes deben dirigirse a un consumidor más crítico y con un mayor manejo de las herramientas comunicativas que lo convierten en un prosumidor. Se trata de un ejercicio complejo realizado quizá en el momento en el que se están produciendo los cambios más profundos en el sector.

\section{Palabras clave}

Estrategia; publicidad; Nueva teoría estratégica; eficacia; crisis; marca; prosumidores.
Fecha de recepción: 01/10/2014

Fecha de revisión: 03/12/2014

Fecha de preprint: 03/12/2014 Fecha de publicación final: 30/12/2014

\begin{abstract}
The present work of investigation to try to realize a revision for the changes that they could have produced in the processes of strategic advertising planning. For this end there has been realized a follow-up of the principal communicative trends in this area, trying to identify the current models to be able this way, to compare the current reality with what it was happening before the crisis. The point of item in this case is in the fact that, as it was foreseeing the New Strategic Theory, the recovery of the human being is the base of the functioning of the advertising current strategy, where the brands must adapt to the preferences of the consumer of an almost individual form, and the messages must go to a more critical consumer and with a major managing of the communicative tools that turn it into a prosumer. It is a question of a complex exercise realized probably in the moment in which the deepest changes are taking place in the sector.
\end{abstract}

\section{Keywords}

Strategic; advertising; New Strategic Theory; efectiveness; crises; brand; prosumers. 


\section{Presentación. Objeto de estudio, hipótesis de partida y metodología}

El presente trabajo forma parte de la línea de investigación sobre estrategia publicitaria desarrollada en la Facultad de Ciencias Sociales y de la Comunicación de la Universidad de Vigo desde el año 2006. Dicha línea ha evolucionado en tres ámbitos de trabajo paralelos: el primero es el relativo a la propia configuración teórica de la disciplina estratégica, desde una perspectiva histórica (Torres-Romay, 2007) y conceptual; un segundo ámbito funciona como observatorio de las tendencias en la aplicación de estrategias publicitarias (López de Aguileta y Torres-Romay, 2007), especialmente en todo lo relativo a las consecuencias de la crisis (TorresRomay, 2010a y 2011); la tercera vía, puesta en marcha de forma simultánea a las anteriores en el año 2010, vincula la estrategia con la eficacia de las campañas, estableciendo posibles correlaciones que den lugar a modelos estratégicos avanzados (Torres-Romay, 2010b). En todo este proceso, dejamos patente la importancia de analizar esta evolución ya que supone mantener el foco en el aspecto más básico del sector publicitario. Así, este trabajo es imprescindible para identificar, mediante un análisis científico, la realidad actual de las estrategias publicitarias y su vinculación con las marcas.

Es precisamente en lo relativo al segundo ámbito - la observación de las tendencias en estrategia publicitaria - donde insertamos esta aportación, si bien el objeto de estudio lleva aparejadas cuestiones vinculadas a la conceptualización del fenómeno estratégico. De esta forma, podemos concretar el objetivo de la investigación en identificar los principales cambios producidos en la aplicación de estrategias publicitarias desde el año 2008 hasta la actualidad, intentado delimitar los mismos en lo relativo a los sujetos del proceso publicitario: anunciantes, agencias, medios y públicos. La acotación temporal se establece desde el estallido de la crisis económica hasta el momento actual, englobando el periodo de mayor descenso de la inversión publicitaria en nuestro país.

El análisis concreto se sustenta en una serie de hipótesis de partida:

- El proceso de planificación estratégica publicitaria parece no haber variado en cuanto a su formulación estructural. De esta forma, sigue existiendo una subordinación de estrategias que condiciona el diseño de estrategias publicitarias concretas.

- La crisis económica no ha supuesto una variación de los procesos estratégicos si no que los cambios producidos son consecuencia de una evolución natural del mercado. De esta forma, las relaciones comerciales plantean un nuevo escenario que debería condicionar las decisiones estratégicas en un proceso de comunicación comercial.

- La valoración desarrollada a lo largo de los años permite confirmar la validez de las estrategias publicitarias clásicas si bien estas caminan de forma paralela a la evolución de la propia disciplina estratégica y, por supuesto, a la adaptación de la estrategia de marketing al contexto comercial actual.

- El planteamiento de la Nueva Teoría Estratégica, formulada en el año 2001, aporta una serie de preceptos vinculados con la recuperación del ser humano, la relación y la articulación social en torno a la estrategia así como una orientación más dialogante, negociadora, cooperativa y consensual que coincide con las tendencias existentes en lo relativo a la situación actual de las estrategias publicitarias.

- En base al contexto descrito - crisis económica, estrategias publicitarias clásicas y Nueva Teoría Estratégica - los sujetos del proceso publicitario han reaccionado de forma desigual:

- Los anunciantes han pasado de la gestión de marca (brand management) a su adaptación, caminando hacia la individualización y personalización de los productos. 
- Las agencias de publicidad se enfrenta, por tanto, a la demanda de unos servicios cada vez más dependientes del conocimiento "personal" de los públicos, creando mensajes que se sustentan en los insights del consumidor y no en los atributos del producto.

- Los cambios en el consumo de medios son, sin duda, los más llamativos con el paso de las audiencias cuantitativas a la valoración de los consumos personalizados y, por lo tanto, la pérdida de peso de los medios masivos convencionales

- La evolución en lo referido al público es la más profunda; el consumidor ha dejado de ser pasivo para convertirse activo y, por tanto, con más control sobre el proceso.

- Como consecuencia de todo lo aportado la eficacia de las estrategias publicitarias depende, claramente, de la correcta aplicación de lo planteado por la Nueva Teoría Estratégica evolucionando desde la marca impersonal al ser humano crítico e independiente.

Para llevar a cabo esta investigación se aplicado una metodología en tres fases. En la primera se realizó un estudio bibliográfico y hemerográfico (según lo recogido en el apartado de bibliografía de este artículo) con la intención de recopilar cualquier propuesta sobre el objeto de estudio en el periodo de acotación del trabajo. En la segunda fase se procedió a un estudio cuantitativo basado en los datos extraídos de las principales bases de datos del sector (citadas en el texto). Estas cifras permiten contextualizar la situación y valorar los cambios que anunciantes, agencias, medios y públicos han aplicado. La tercera fase pasa por un análisis cualitativo de los recursos conseguidos en las dos fases anteriores identificando las tendencias e intentado reconocer modelos concretos.

En cuanto a la estructura del trabajo, establecemos tres bloques básicos: el primero se refiere al concepto, el segundo al contexto y el tercero al análisis del objeto de estudio específico. Como se podrá comprobar no atendemos a las fases de investigación ya que la puesta en marcha de cada una de ellas es muy minuciosa y su síntesis para este artículo resulta fundamental.

\section{Delimitación del concepto de estrategia vinculado a la comunicación}

Los orígenes del concepto estrategia se remontan siglos atrás. La mayor parte de los escasos manuales existentes sobre estrategias de comunicación inician su repaso por la historia de esta disciplina aludiendo a los orígenes militares del término: "la conducta estratégica es tan antigua como el hombre. El concepto de estrategia ha existido siempre - cualquiera que haya sido el término utilizado - para significar la necesidad de seguir ciertas reglas en la dirección de los asuntos que permitan optimizar los recursos y las fuerzas a utilizar" (Pérez, 2001: 25). Sin embargo, no fue hasta el siglo XX que la estrategia fue abordada desde un paradigma lógico/formal o científico (que superaba el paradigma militar anterior) a través de desarrollos como la Teoría de los Juegos formulada por Morgersterm y Neuman (1944).

A mediados del siglo pasado la estrategia alcanza un gran auge debido a su aplicación a través del management lo que provocó que en las décadas siguientes casi toda la innovación de la estrategia se genere en las Business Schools, con los consiguientes sesgos y vicios de origen estableciendo un paradigma económico/directivo. La primera gran ruptura de esa tendencia se produce en 1987 cuando Itami pone de relieve la importancia de los intangibles que, hasta entonces, habían sido olvidados por los economistas. Cobran importancia, entonces conceptos como "diferenciación/imagen" o "cultura/comunicación corporativa". La segunda ruptura la integran las distintas aportaciones en torno a la "gestión del conocimiento" y la "organización que aprende". Al introducir la Teoría General de Sistemas en el campo del management y en el de Teoría de la Organización se provoca un gran cambio en la forma de entender las relaciones de las corporaciones con su entorno y, en consecuencia, la manera de plantear sus estrategias. 
En 1994 un grupo de autores, liderados por Prahalad y Hamel, se atreven a cuestionar el paradigma vigente abriendo un complejo debate sobre la estrategia. El management no era cuestionado como tal pero si era acusado de tener claros sesgos: el enfoque económico, el uso de variables instrumentales, su matematismo, instrumentalismo, funcionalismo...

A partir del año 2000 el desarrollo de la Nueva Teoría Estratégica abordó ciertos problemas seculares, añadiendo un nuevo paradigma, el humano relacional (Pérez y Massoni, 2009). Según se recoge en la síntesis de esta nueva teoría, son cinco los aspectos fundamentales de la estrategia actual:

"1) (...) la clave de todo teoría estratégica pasa por recuperar al ser humano (...)

2) (...) la estrategia como ciencia de la relación y la articulación social (...)

3) (...) una orientación más dialogante, negociadora, cooperativa y consensual.

4) Añadiendo a los factores económicos - que siempre estarán ahí - otros más relacionales e intangibles.

5) En vez de preocuparse por proporcionar reglas paradigmáticas y exactas la "nueva teoría estratégica" aspira a proporcionar reglas hermenéuticas, orientativas y articuladoras"(NTE, 2014).

Conociendo, por tanto, la evolución del concepto de estrategia, debemos dar un paso más centrándonos en lo relativo a las estrategias de comunicación. Desde ese punto de vista, el tema que nos ocupa se caracteriza por la inexistencia de trabajos académicos específicos de amplitud, limitándose a aportaciones escasas o aspectos puntuales. De una forma más concreta, la estrategia publicitaria tampoco cuenta con un bagaje significativo en cuanto a la investigación académica en nuestro país, siendo objeto de publicaciones científicas concretas y poco habituales (Fernández, Alameda y Martín, 2011), algo lógico sobre todo si tenemos en cuenta la rápida y confusa evolución del fenómeno.

El caso que nos ocupa, el desarrollo evolutivo en la aplicación de la estrategia a la publicidad, se basa en procesos de gestión (management) en base a los intangibles buscando resultados en términos de eficacia económica y comunicacional. Surge así un proceso de planificación estratégica publicitaria que pasa por diversas fases sustentadas en los principios de investigar, decidir y actuar.

Durante la investigación llevada a cabo en los últimos años y tras la valoración de casos prácticas y conocimiento de desarrollos teóricos podemos llegar a sintetizar una serie categorías que agrupan las distintas fórmulas estratégicas (Torres-Romay, 2012):

a) Estrategias de posicionamiento. Se trata de unas estrategias que han ido adquiriendo cada vez más relevancia para las empresas. Esta afirmación se refleja en una mayor utilización del posicionamiento como herramienta para competir en un ambiente ampliamente globalizado. Conscientes de esta situación a lo largo de la historia la literatura académica ha ido desarrollando diferentes perspectivas de las estrategias dentro de esta vía de trabajo (Torres y Muñóz, 2006).

Uno de los elementos que se deben destacar en este tipo de estrategias es que incluye aspectos que no están relacionados directamente con el producto o con los gustos y preferencias de los consumidores, sino de una forma más específica con la cultura del consumidor. De hecho, esta estrategia considera que la publicidad realizada por las diferentes marcas varía según la cultura que caracteriza a la región a la cual va dirigida (Aaker y Maheswaran, 1997). 
En cualquier caso, siguiendo a Michael Porter (1980), el posicionamiento podría determinarse en función de dos parámetros básicos: decidir si se quiere ser líder en coste o en diferenciación y decidir si quiere hacerse para todo el mercado o para un nicho concreto. Las posibles combinaciones dan lugar a las distintas estrategias de posicionamiento.

b) Estrategias competitivas. Su objetivo es quitarle ventas a la competencia, convirtiendo a sus clientes en propios, generando conocimiento de su oferta o induciendo a la prueba. Dentro de estas podemos contemplar varias subcategorías:

\section{b.1. Estrategias comparativas: muestran las ventajas frente a la competencia.}

b.2. Estrategias financieras: se basan en una presencia en la mente de los destinatarios superior a la de la competencia, acaparando el espacio publicitario.

b.3. Estrategias promocionales: en las que se busca mantener o incrementar el consumo del producto, contrarrestar alguna acción de la competencia o incitar a la prueba del producto. Dentro de esta se recurre a estrategias de empuje (push) para motivar en los puntos de venta a través de los distribuidores o fuerzas de venta, y de tracción (pull) para estimular al consumidor final.

b.4. Estrategias de imitación: que consisten en imitar lo que hace el líder o la mayoría de los competidores.

c) Estrategias de desarrollo. Su finalidad es potenciar el crecimiento de la demanda, aumentar el número del cliente. Esto se consigue mediante varias fórmulas:

c.1. Estrategias extensivas: que buscan conquistar a nuevos consumidores.

c.2. Estrategias intensivas: que pretenden que los clientes consuman más.

d) Estrategias de fidelización. Pueden considerarse complementarias de las anteriores. Tratan de retener a los consumidores del producto y mantener su fidelidad de comprar y consumo. Es decir, conseguir un mercado cautivo que garantice las ventas futuras con las correspondientes evoluciones del precio.

Entendemos, por tanto, que la evolución de la disciplina estratégica conjugada con la concepción de esta como un elemento básico de la gestión ha llevado a la creación de estos modelos, que sintetizamos en la siguiente tabla.

Tabla 1. Estrategias publicitarias clásicas

\begin{tabular}{|c|c|l|}
\hline \multirow{4}{*}{$\begin{array}{c}\text { Competitivas. } \\
\text { Su objetivo es quitarle ventas } \\
\text { a la competencia. }\end{array}$} & Comparativas & Enseñar las ventajas del producto frente a la competencia. \\
\cline { 2 - 3 } & Financieras & Presencia mayoritaria en las mentes de los consumidores. \\
\cline { 2 - 3 } & Posicionamiento & Darle a la marca un lugar en la mente del consumidor. \\
\cline { 2 - 3 } & Imitación & Imitar la estrategia de la competencia. \\
\cline { 2 - 3 } $\begin{array}{c}\text { Desarrollo. } \\
\text { Potenciar el crecimiento de la } \\
\text { demanda }\end{array}$ & Extensivas & Ampliar la demanda en nuevos sectores. \\
\cline { 2 - 3 } $\begin{array}{c}\text { Fidelización } \\
\text { Intensivas }\end{array}$ & Ampliar la demanda entre los clientes actuales. \\
\hline
\end{tabular}

Fuente: elaboración propia 


\section{Situación actual del sector publicitario en España}

Una vez delimitada la evolución del concepto y establecer una síntesis básica de las principales estrategias publicitarias existentes, debemos intentar conocer los avances en lo referido a esas estrategias en nuestro país. Para ello, y como paso previo, debemos aproximarnos a la situación del sector publicitario desde la perspectiva cuantitativa y cualitativa, intentando identificar cómo se aborda el proceso de planificación estratégica en la actualidad.

\subsection{Los datos del sector publicitario}

El sector publicitario español arrastra pérdidas desde el inicio de la crisis (Torres-Romay, 2012), tal y como podemos observar en la gráfica 1. Todos los analistas coinciden en que aunque se produjera una recuperación económica, resultará imposible regresar a los niveles anteriores (Legerén y García-Mirón, 2012). En este contexto, el sector publicitario ha tomado una serie de decisiones para mantenerse a flote, alguna de ellas correctas pero otras claramente discutibles (Torres-Romay, 2009).

\section{Gráfico 1. Evolución de la inversión publicitaria en España}

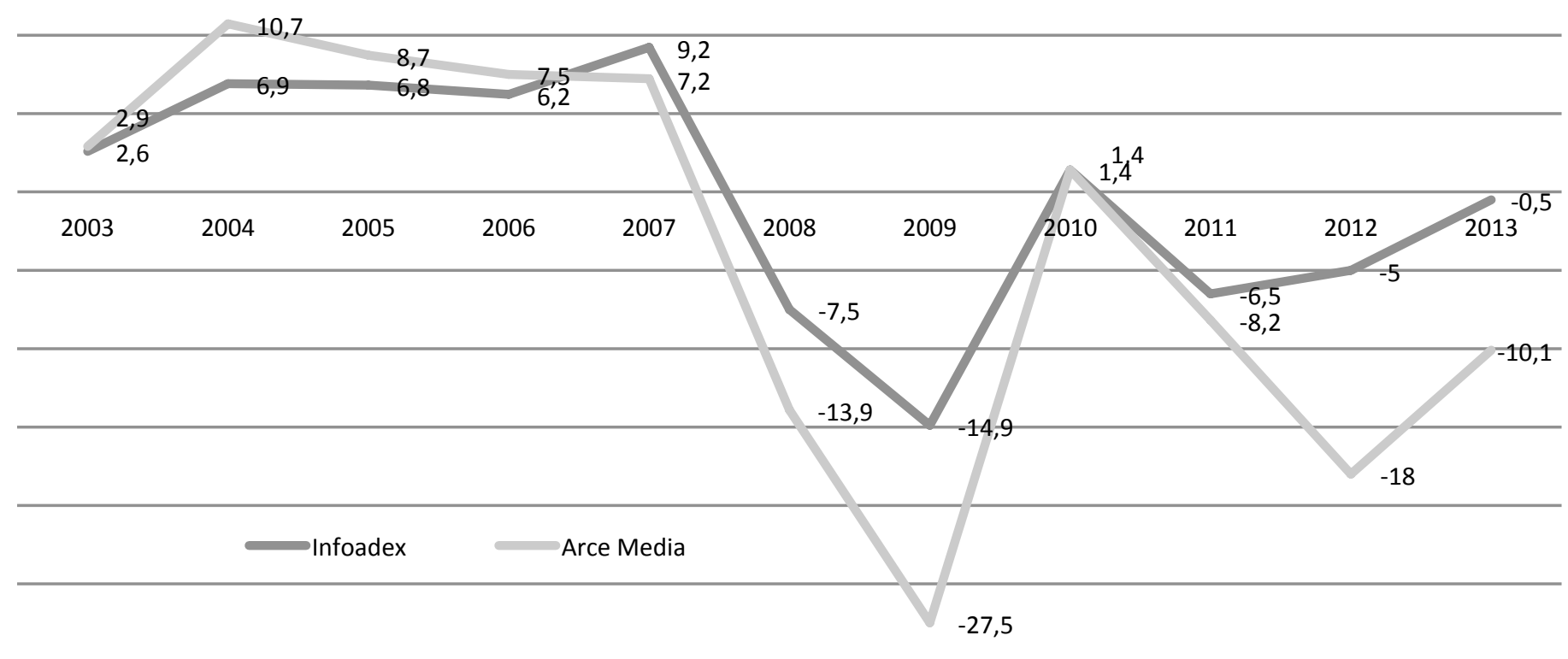

Fuente: Infoadex y Arce Media, 2014

Hasta el año 2007 la publicidad acumulaba cinco años de incremento de la inversión llegando a subidas de más del nueve por ciento. Esta evolución se producía en un contexto de estabilidad o incluso de "bonanza" económica (Narayadou, 2006) en el que no se preveían los problemas que finalmente se han producido. Bien es cierto que la realidad de los datos nos habla de que el receso publicitario se ha producido con cierta lentitud. La crisis se hizo visible en el sector publicitario en el cuarto trimestre de 2008, debido sobre todo a la caída de la inversión de anunciantes especialmente vulnerables a esta coyuntura, como los del sector inmobiliario y los de las marcas de automóvil (Infoadex, 2009). 
En este contexto queremos conocer cómo se pone en marcha el proceso de planificación estratégica y si este puede haber sufrido modificaciones como consecuencia de la crisis.

\subsection{El proceso estratégico publicitario}

Desde un punto de vista teórico, el diseño de estrategias publicitarias responde a un modelo relativamente estable. Según la estructura recogida por García Úceda (2005) el proceso se inicia en la empresa con la puesta en marcha de la estrategia general que incluye, entre otras la estrategia comercial o de marketing donde se toman decisiones sobre los parámetros de venta tanto desde una perspectiva estratégica como operativa (precio, producto, distribución y comunicación). El diseño del mix de comunicación puede implicar la puesta en marcha de campañas publicitarias, que contarán con su diseño estratégico específico.

Es en este punto donde debemos retomar lo aportado por Pérez (1996) en lo relativo a la subordinación de estrategias. De esta forma, las decisiones de carácter empresarial, tomadas desde la dirección de la empresa, necesariamente condicionarán la estrategia de marketing que, a su vez, influirá en la estrategia publicitaria y, por tanto, en la estrategia creativa - el propio mensaje (Torres-Romay, 2005) - y de medios de una campaña.

Si aplicamos el concepto de estrategia desde la perspectiva de Ansoff, Declerck y Hayes (1976) debemos entender que estrategia es la "dialéctica de la empresa con su entorno". De esta forma las empresas deberían centrarse en tomar decisiones que respondan estratégicamente a las nuevas necesidades de su mercado. El resultado desde el punto de vista comunicativo, no es el mismo en todos los casos y depende en buena medida del análisis que la empresa haga del mercado y de la estrategia que diseñe para hacerle frente.

En el momento actual, nos encontramos con que las empresas han adoptado una política de austeridad que afecta a todos los departamentos. Desde el punto de vista del marketing podemos podemos aportar datos específicos sobre cada uno de los parámetros operativos, denominados las 4 pés del marketing: producto, precio, distribución y comunicación (TorresRomay, 2010c). En lo referido al producto, los recortes aplicados en todos los departamentos incluyen los gastos de I+D+i, lo que supone que a partir del año 2009 se redujeron notablemente los lanzamientos de nuevos productos. De esta forma, los ejes de producto que habían conseguido una notable presencia en los años anteriores han sido, poco a poco, abandonados en favor de otras líneas de trabajo.

En cuanto a la distribución, en este momento nos estamos enfrentando a un cambio importante. Atendiendo a la evolución del modelo de consumo, derivado de la crisis económica, muchas de las principales cadenas de distribución han tomado decisiones estratégicas que afectan profundamente a la relación de fuerzas del mercado. De esta forma se está produciendo una consolidación de las denominadas "marcas blancas" o marcas de distribución en detrimento de las marcas comerciales que tradicionalmente realizaban un mayor esfuerzo publicitario. La tendencia se extiende de los distribuidores a todo tipo de sectores y es, hoy por hoy el principal reto de las marcas fabricantes. Quizá relacionado con el fenómeno anterior, el precio es la principal variable en la que parecen estar trabajando las empresas. La reducción de los precios se ha convertido en uno de los principales recursos para las marcas. De esta forma la bajada de los precios de los productos unida al descenso de la inversión en comunicación, configura un panorama publicitario complejo en el que la estrategia promocional parece la única posible.

Todo lo anterior dibuja el contexto bajo el cual debe situarse la estrategia publicitaria por lo que lo que debemos hacer a continuación es proceder al análisis de los sujetos que están implicados en este proceso para hacer frente así al objeto de esta investigación. 


\section{Los sujetos del proceso publicitario desde la perspectiva estratégica}

\subsection{La gestión de la marca por parte de los anunciantes}

Durante el año 2008 el número de empresas activas en nuestro país ha descendido notablemente, situándose además como uno de los de menor índice de creación de nuevas empresas a nivel mundial. Todos estos datos se imbrican en un contexto social, político y económico en el que el empresariado puntúa muy bajo la calidad de las instituciones públicas y económicas, estando la percepción empresarial entre las más negativas de europea.

En lo referido específicamente a los anunciantes los datos aportados en la tabla recogen ese pesimismo instalado en el sector empresarial. Las reducciones son profundas aunque los principales anunciantes sigan siendo los mismos, con la clara excepción de la desaparición de las marcas de automóviles (Torres-Romay, 2012).

Tabla 2. Evolución del ranking de anunciantes en España

\begin{tabular}{|c|c|c|c|c|c|c|c|c|c|c|c|c|c|c|c|c|c|c|c|c|}
\hline \multirow[b]{2}{*}{ Anunciantes } & \multicolumn{2}{|c|}{2013} & \multicolumn{2}{|c|}{2012} & \multicolumn{2}{|c|}{2011} & \multicolumn{2}{|c|}{2010} & \multicolumn{2}{|c|}{2009} & \multicolumn{2}{|c|}{2008} & \multicolumn{2}{|c|}{2007} & \multirow{2}{*}{$\begin{array}{l}\% \text { incr. } \\
(07-08)\end{array}$} & \multirow{2}{*}{$\begin{array}{l}\% \text { incr. } \\
(08-09)\end{array}$} & \multirow{2}{*}{$\begin{array}{l}\% \text { incr. } \\
(09-10)\end{array}$} & \multirow{2}{*}{$\begin{array}{c}\% \\
\text { incr. } \\
(10- \\
11)\end{array}$} & \multirow{2}{*}{$\begin{array}{c}\% \\
\text { incr. } \\
(11- \\
12)\end{array}$} & \multirow{2}{*}{$\begin{array}{c}\% \\
\text { incr. } \\
(12- \\
13)\end{array}$} \\
\hline & Rk & Valor & RK & Valor & RK & Valor & RK & Valor & RK & Valor & RK & Valor & RK & Valor & & & & & & \\
\hline $\begin{array}{c}\text { Telefónica } \\
\text { S.A.U. }\end{array}$ & 5 & 56,1 & 4 & 67,4 & 3 & 113,4 & 2 & 131,0 & 1 & 129,3 & 1 & 173,8 & 1 & 171,2 & 1,5 & $-25,2$ & $-1,8$ & $-13,4$ & $-40,5$ & $-16,7$ \\
\hline $\begin{array}{c}\text { Procter \& } \\
\text { Gamble } \\
\text { España S.A. }\end{array}$ & 1 & 122,4 & 1 & 105,1 & 2 & 123,8 & 3 & 132,2 & 2 & 111,4 & 2 & 131,9 & 2 & 146,6 & $-10,0$ & $-12,6$ & 16,8 & $-6,3$ & $-15,1$ & 16,4 \\
\hline $\begin{array}{c}\text { L'Oreal } \\
\text { España S.A. }\end{array}$ & 2 & 79,4 & 3 & 79,6 & 4 & 92,2 & 5 & 105,5 & 3 & 97,0 & 3 & 107,7 & 3 & 121,1 & $-11,1$ & $-9,8$ & 6,5 & $-12,6$ & $-13,6$ & $-0,2$ \\
\hline $\begin{array}{c}\text { El Corte } \\
\text { Inglés S.A. }\end{array}$ & 3 & 76,8 & 2 & 86,9 & 1 & 171,3 & 1 & 174,3 & 4 & 90,0 & 4 & 96,4 & 4 & 110,1 & $-12,4$ & $-4,3$ & 9,5 & $-1,7$ & $-49,2$ & $-11,6$ \\
\hline $\begin{array}{l}\text { Vodafone } \\
\text { España S.A. }\end{array}$ & 4 & 63,4 & 5 & 61,6 & 7 & 59,7 & 6 & 77,6 & 6 & 71,7 & 5 & 87,5 & 6 & 80,9 & 8,2 & $-22,2$ & $-4,4$ & $-23,0$ & $-3,2$ & 3,7 \\
\hline $\begin{array}{l}\text { Volkswagen- } \\
\text { Audi España }\end{array}$ & 6 & 56,0 & 6 & 61,1 & 5 & 85,1 & 4 & 111,5 & 5 & 73,5 & 6 & 86,6 & 5 & 99,5 & $-13,0$ & $-28,2$ & 4,7 & $-23,7$ & $\mid-28,2$ & $-8,3$ \\
\hline $\begin{array}{c}\text { Renault } \\
\text { España } \\
\text { Comercial } \\
\text { S.A. }\end{array}$ & 18 & 25,6 & 14 & 31,0 & 14 & 34,5 & 16 & 36,3 & 20 & 32,3 & 7 & 69,7 & 7 & 74,2 & $-6,0$ & $-53,3$ & 14,5 & $-4,9$ & $-10,1$ & $-17,4$ \\
\hline Danone S.A. & 10 & 41,7 & 8 & 41,3 & 9 & 50,0 & 9 & 59,9 & 7 & 61,2 & 8 & 67,7 & 10 & 70,1 & $-3,6$ & $-9,1$ & $-8,0$ & $-16,5$ & $-17,4$ & 0,9 \\
\hline $\begin{array}{l}\text { ING Direct, } \\
\text { Suc.España }\end{array}$ & 7 & 47,0 & 7 & 57,8 & 8 & 57,1 & 11 & 44,6 & 8 & 56,6 & 9 & 61,4 & 8 & 72,5 & $-15,2$ & $-6,3$ & $-15,2$ & 27,3 & 0,2 & $-18,6$ \\
\hline $\begin{array}{c}\text { France } \\
\text { Telecom } \\
\text { España, S.A. }\end{array}$ & 9 & 42,6 & 11 & 39,3 & 10 & 49,5 & 7 & 71,7 & 10 & 45,6 & 10 & 58,4 & 9 & 47,7 & $-19,5$ & $-21,5$ & 26,3 & $-31,1$ & $-20,6$ & 8,3 \\
\hline
\end{tabular}

Fuente: Infoadex, 2014. 
La reducción de los presupuestos resulta evidente y especialmente grave en algunos casos, pero los datos deben ser valorados de una forma más profunda. Es necesario intentar establecer algún tipo de correlación entre la inversión publicitaria y el valor de la marca. Partimos de la existencia de una gestión de marca (brand management) que se había tomado como base en el periodo anterior al estallido de la crisis. El concepto de marca se relaciona, en el ámbito de la publicidad, con dos ideas fundamentales: la imagen y la identidad.

La imagen de marca es algo intangible pero que sirve para que una determinada empresa comunique su cultura empresarial y cree una determinada marca, logotipo e identidad corporativa. Toda marca tiene una determinada imagen, que por medio de su eficiente planificación y control puede convertirse en una eficaz estrategia de comunicación que apoya en todo momento y a cada uno de los productos. En definitiva, esa imagen global es el resultado de la política integrada y de una gestión eficaz de los procedimientos, medios y oportunidades de comunicación, o sea, comunicación que se basa en la marca y en la identidad corporativa. No debemos, en cualquier caso, confundir la marca (como signo gráfico/verbal) con la imagen que se transmite a través de la publicidad y la presentación de los productos (imagen de marca).

La identidad de la marca es el conjunto de activos vinculados al nombre al nombre el símbolo de la marca que incorpora el valor suministrado por un producto o servicio, la compañía o sus clientes. Las principales categorías de activos son: 1) El reconocimiento del nombre de la marca; 2) la fidelidad a la marca; 3) la calidad percibida; 4) Las asociaciones con la marca.

Una vez delimitadas las diferencias entre imagen e identidad, es preciso tener presente que cada activo de la marca crea valor de forma diferente. Por lo tanto, para gestionar efectivamente el valor de la marca y para tomar decisiones sobre actividades de construcción de marca es importante que el responsable sea sensible a la forma por las que las marcas crean valor tanto para el cliente como para la compañía. Hasta hace 25 años el concepto de marca era conocido pero apenas "gestionado" ni siquiera en el área de marketing. En cambio ahora la marca interesa no sólo a los responsables de marketing, sino también a financieros, auditores, analistas y expertos fiscales. El método de medición y valoración de las marcas más aceptado internacionalmente es el de la agencia Interbrand. Tomando este como referencia, podemos cruzar los datos con los de inversión publicitaria en España, obteniendo la tabla 3.

De esta forma, lo que pretendemos valorar es si los esfuerzos de inversión publicitaria tienen algún tipo de repercusión en el valor de la marca. Como punto de partida podemos indicar que nueve de los diez principales anunciantes de nuestro país tienen sus marcas posicionadas en los "tops" de valor de marca de Interbrand bien a nivel nacional (Mejores marcas españolas), a nivel regional-Europa (Eurobrand) o global (Best Global Brands). Por otra parte podemos establecer una correlación parcial entre el descenso de la inversión publicitaria y la reducción del valor de la marca, si bien en este caso se trata de un análisis mucho más prudente limitado a aquellos casos donde se analiza el impacto de la inversión publicitaria en España y se cuenta con una valoración global de la marca. El ejemplo más paradigmático a este respecto es el de Telefónica, una de las compañías que ha visto reducida su inversión publicitaria prácticamente a la mitad desde 2008 y que contempla como se desploman los valores de sus marcas en España y en Europa. Algo semejante sucede con el Corte Inglés mientras que en lado opuesto encontramos marcas como Volkswagen o Danone, recortando sus presupuestos de inversión nacional pero consiguiendo mejoras de su valoración a nivel Europeo o internacional.

Dando por válida la premisa de la influencia de la inversión publicitaria (y por tanto de la estrategia publicitaria) en el valor de la marca, el siguiente paso es entender cómo se gestiona esa estrategia para contribuir al mantenimiento o revalorización de la marca algo que, en términos generales, también es responsabilidad de las agencias de publicidad. 
Tabla 3. Relación inversión publicitaria / Valor de marca

\begin{tabular}{|c|c|c|c|c|c|c|c|c|}
\hline \multirow[b]{2}{*}{ Anunciante } & \multicolumn{4}{|c|}{ INVERSIÓN PÚBLICITARIA 2013} & \multicolumn{4}{|c|}{ VALOR DE MARCA 2013} \\
\hline & Marca & RK & $\begin{array}{c}\text { Inv. } \\
\text { (mil.€) }\end{array}$ & $\begin{array}{c}\text { Var. (\%) } \\
12-13 \\
\end{array}$ & RK & $\begin{array}{l}\text { Valor } \\
\text { (mil.) }\end{array}$ & \begin{tabular}{|c|}
$\operatorname{Var}(\%)$ \\
$12-13$ \\
\end{tabular} & Fuente \\
\hline Procter \& Gamble España & $P \& G$ & 1 & 122,4 & $-16,7$ & 7 & $47.445 €$ & 0,6 & Eurobrand 2013 \\
\hline \multirow{2}{*}{ L'Oreal España S.A. } & L'Oreal & \multirow{2}{*}{2} & \multirow{2}{*}{79,4} & \multirow{2}{*}{16,4} & 39 & $9.874 \$$ & 12,0 & Best Global Brands, 2013 \\
\hline & L'Oreal & & & & 69 & $12.018 €$ & 9,4 & Eurobrand 2013 \\
\hline El Corte Inglés S.A. & El Corte Inglés & 3 & 76,8 & $-0,2$ & 10 & $889 €$ & $-35,0$ & Mejores marcas españolas, 2013 \\
\hline Vodafone España S.A. & Vodafone & 4 & 63,4 & $-11,6$ & 18 & $28.926 €$ & 1,2 & Eurobrand 2013 \\
\hline \multirow{3}{*}{ Telefónica S.A.U. } & Movistar & \multirow{3}{*}{5} & \multirow{3}{*}{56,1} & \multirow{3}{*}{3,7} & 1 & $11.455 €$ & $-31,0$ & \multirow{2}{*}{ Mejores marcas españolas, 2013} \\
\hline & Telefónica & & & & 17 & 553 & $-56,0$ & \\
\hline & Telefónica & & & & 36 & $18.083 €$ & $-17,8$ & Eurobrand 2013 \\
\hline \multirow{3}{*}{ Volkswagen-Audi España } & Volkswagen & \multirow{3}{*}{6} & \multirow{3}{*}{56,0} & \multirow{3}{*}{$-8,3$} & 34 & $11.120 \$$ & 20,0 & Best Global Brands, 2013 \\
\hline & Volkswagen & & & & 6 & $23.121 €$ & s.d & Eurobrand 2013 \\
\hline & Audi & & & & 51 & $7.767 \$$ & 8,0 & Best Global Brands, 2013 \\
\hline ING Direct, NV. Suc. España & ING & 7 & 47,0 & $-17,4$ & & s.d & s.d & s.d \\
\hline France Telecom España, S.A. & Orange & 9 & 42,6 & 0,9 & 2 & $19.851 €$ & s.d & Brand Finance France 2014 \\
\hline \multirow{2}{*}{ Danone S.A. } & Danone & \multirow{2}{*}{10} & \multirow{2}{*}{41,7} & \multirow{2}{*}{$-18,6$} & 49 & $7.968 \$$ & 6,0 & Best Global Brands, 2013 \\
\hline & Danone & & & & 80 & $10.748 € €$ & 1,2 & Eurobrand 2013 \\
\hline Renault España Comercial & Renault & 18 & 25,6 & 8,3 & 10 & $9.010 €$ & s.d & Brand Finance France 2014 \\
\hline
\end{tabular}

Fuente: Infoadex e Interbrand, 2014.

\subsection{El papel de las agencias publicitarias en la creación de estrategias y mensajes}

Desde la perspectiva de las agencias de publicidad, existe una tendencia a considerar que existe una crisis permanente en el sector. Por diversos factores, o quizá por una tendencia autocrítica muy acuciada, en foros profesionales se habla de crisis publicitaria como si de un mal endémico se tratara (Pérez Ruíz, 2001). De hecho, desde una perspectiva económica, las recesiones producidas a lo largo de la historia afectaron claramente a las cifras de negocio nacional e internacional (Osio, 2008). En los años noventa se hablaba de la crisis de la publicidad en términos de crisis inversora, por la reducción de las inversiones publicitarias; crisis comunicativa, por la falta de eficacia de la publicidad derivada de la saturación de los medios convencionales y, por supuesto, crisis de las agencias de publicidad, al no adecuarse su modelo de funcionamiento a las necesidades del momento (Benavides, 1993).

Con independencia de los datos, las relaciones entre las agencias y los anunciantes parecen no haber variado excesivamente como consecuencia de la crisis. Los clientes siguen demandando de los publicitarios: creatividad, buen servicio, conocimiento del mercado y decisión estratégica y el porcentaje de satisfacción con su agencia resulta alto (Grupo Consultores, 2012).

Pero más allá de la consecuencias de la crisis, el interés en este punto se centra en establecer una relación entre lo aportado hasta ahora (inversión publicitaria y valor de marca) y la estrategia publicitaria. Teniendo en cuenta los condicionantes establecidos en este estudio especialmente en lo relativo a la variación de la estrategia de marketing - las principales agencias de publicidad del país se enfrentan a un reto complejo ya que dentro del desarrollo de estrategias publicitarias clásicas (tabla 1) resulta evidente que las estrategias competitivas de carácter promocional, destinadas a mantener o incrementar el consumo, son el recurso más obvio. 
Realizando un proceso de briefing podemos valorar si la afirmación anterior resulta correcta mediante el análisis de contenido de las campañas publicitarias de los principales anunciantes del país. En la investigación desarrollada en 2009 se recogieron campañas emitidas durante el primer semestre de ese año estableciendo una clasificación de las mismas en función del tipo de mensaje. Se crearon tres categorías:

a) Adaptación de mensajes preexistentes. Se trata de piezas publicitarias de campañas anteriores, adaptando alguno de sus elementos secundarios (off, texto, elementos gráficos...) a un mensaje acorde con la nueva estrategia planteada. Es un recurso que ha sido utilizado mucho durante los años 2009 a 2012, especialmente por las marcas de automóviles que reponían piezas anteriores haciendo hincapié en las promociones y facilidades de pago propias o derivadas de las ayudas del gobierno.

b) Creación de mensajes adaptados al contexto. Otra tendencia observada en la investigación de 2009 es la de crear mensajes en los que la realidad de la crisis aparece presente de forma clara. Es lo que las publicaciones del sector han denominado "Advertcrisis" y que ha dado lugar a piezas publicitarias con narrativas emotivas y humanas en las que se hablaba directamente de la crisis y sus consecuencias insertando al producto en la realidad social del momento.

c) Creación de mensajes con estrategia independiente. En el periodo más grave de la crisis (2009-2012) resultaba complejo localizar campañas que aplicaran estrategias de lanzamiento de nuevos productos. Vinculado con esto, el lanzamiento de nuevas líneas creativas derivadas de estrategias novedosas resulta cada vez más escasa. Se trata de una afirmación que hay que matizar ya que existen piezas publicitarias que no se adscriben a los grupos anteriores pero que tampoco se pueden considerar una innovación ya que mantienen una línea similar a lo que la marca ya estaba haciendo.

Sea como fuere, el interés de este trabajo se centra, precisamente en esta última categoría, ya que se trata de la única forma de entender si se están aplicando estrategias que avancen o modifiquen las que hemos considerado clásicas. Desde este punto de vista, podemos volver a tomar como referencia a los principales anunciantes por inversión publicitaria, si bien debemos cuestionar esta opción por el hecho de que una mayor inversión no necesariamente significa una mejor gestión de la estrategia publicitaria, de hecho, análisis anteriores han concluido que las marcas con mayor inversión son las más tradicionales a la hora de plantear sus propuestas comunicativas, siendo más conservadoras y menos arriesgadas.

Para buscar una vía más precisa de valoración de esa nueva creación de estrategias una referencia interesante es la que obtenemos en la lista corta de los Premios de Eficacia. Dentro de la categoría de "eficacia en comunicación comercial" encontramos una interesante combinación de marcas globales (Coca-Cola, Heineken, McDonald's...) y marcas nacionales (Mahou, El Corte Inglés, Campofrío...). En la tabla siguiente recogemos la lista indicando el tipo de estrategia que se aplica en cada campaña: 
Tabla 4. Lista corta premios eficacia 2014 con análisis de estrategias

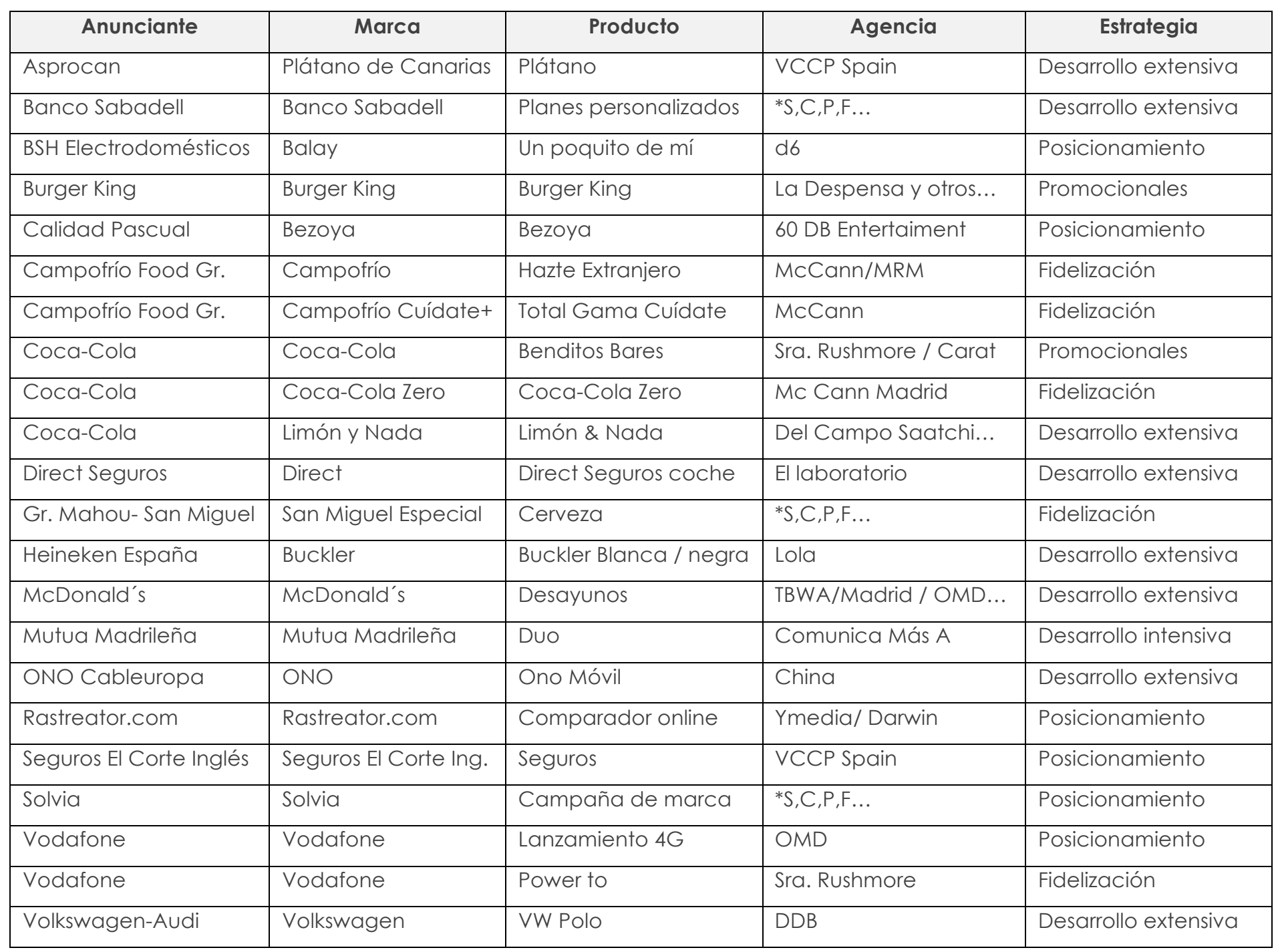

Fuente: Premios Eficacia, 2014.

Aunque el análisis se ajuste tan sólo a la visualización de las piezas, en la mayor parte de los casos la identificación de la estrategia aplicada resultaba sencilla. La valoración general de la tabla deja entrever alguna cuestión ya apuntada - como la importancia de las estrategias de posicionamiento - al tiempo que identificamos un singular esfuerzo por la búsqueda de nuevos mercados. Las estrategias de fidelización también están presentes y no parece casual que esta sea empleada por marcas asentadas como Vodafone, San Miguel o Campofrío.

Debemos profundizar algo más en el análisis realizado ya que lo que diverge de la simple aplicación de las estrategias clásicas es el argumento de venta (reason why) de las campañas. Nos encontramos así que la calidad y la tradición se convierten en elementos básicos de venta, acompañados de referencias locales e incluso individuales. De esta forma, no es casual que Coca-Cola haya emprendido una campaña promocional que tiene como protagonista los bares (un concepto muy español) o que McDonald's presente desayunos según la costumbre española y Campofrío emprenda una campaña hablando de emigración ("Hazte extranjero"). 
Pero el caso más paradigmático resulta ser el de Balay, que ha hecho protagonista de sus campañas a los trabajadores de su fábrica. Se trata de una personalización de la comunicación que completa el círculo de lo que podemos denominar "marcas adaptadas". Mediante una adecuada combinación de los elementos que componen la estrategia desarrollan mensajes (y posicionamientos) que permiten un mayor acercamiento al consumidor tanto por cuestiones geográficas como culturales e identitarias. En este sentido, el trabajo de campo desarrollado ha detectado tres líneas básicas en la adaptación de las marcas:

a) Adaptación cultural: en aquellos casos en los que el mensaje contempla aspectos culturales del lugar donde es difundido, como ha hecho McDonald's incidiendo en que sus materias primas se adquieren en mercados locales. Incluimos en esta categoría aquellas piezas que emplean la identidad de un lugar como ventaja competitiva, tal es el caso de los anuncios de Seat como "coche hecho en España".

b) Adaptación tradicional: aquellas campañas que apelan a la trayectoria de la marca y su vinculación en la historia personal del consumidor. Tal es el caso de Danone que ha pasado de la confrontación directa con las marcas blancas a apelar al recuerdo de sus jingles y campañas de décadas anteriores.

c) Adaptación personal: acciones comunicativas que tienen en cuenta las características individuales de cada uno de los consumidores. La campaña emprendida en 2013 por Coca-Cola permitiendo personalizar las latas o la marca Nutella que facilita etiquetas con el nombre del comprador son ejemplos claros de esta última tendencia.

Las fórmulas anteriores no eliminan el trabajo realizado en cuanto a la valorización de las marcas, sino que se suman a ese esfuerzo aportando ventajas específicas en mercados concretos. La cuestión, en este punto, es determinar si las estrategias publicitarias puestas en marcha por las agencias de publicidad, son difundidas por los medios adecuados.

\subsection{Los cambios en el consumo de medios}

La situación económica de los medios de comunicación no es buena, y son precisamente los malos datos publicitarios los que provocan esa situación que, a su vez, depende de que los principales anunciantes recuperen su capacidad inversora. Sin embargo, la crisis económica está acelerando un proceso que, quizá, ya estaba en marcha antes de que los problemas económicos se presentaran de forma más clara. La batalla entre los denominados medios convencionales y los no convencionales sigue librándose con resultados que apuntan a un panorama complejo para los primeros.

Este cambio supone que los medios convencionales tienen más complicada su financiación a través de la publicidad. Los anunciantes apuestan por los no convencionales y hasta ahora la diferencia oscilaba y no era demasiado notable, pero la caída resulta paulatina y constante (Gráfico 2). Debemos tener en cuenta la posibilidad de que este cambio del panorama mediático tenga una causa en la menor eficacia de los medios convencionales derivada de la evolución de sus audiencias. Es cierto que no se trata de un cambio radical, ya que la televisión, por ejemplo, sigue siendo el "medio rey".

De hecho, el mantenimiento de la publicidad en medios convencionales se sustenta, sin duda, en la importancia social de esos medios. En términos de penetración, la televisión es, con diferencia el medio con mayor índice en nuestro país y todo ello a pesar del rápido crecimiento que internet ha experimentando en los últimos años y a la constancia de la consolidación del fenómeno del consumo "multipantalla" (Marta y Gabelas, 2008) 
De forma paralela a este cambio en las fórmulas de consumo y como consecuencia de los procesos de digitalización (García, 2008) su capacidad de segmentación es cada vez mayor. Tanto la televisión, como el cine o la prensa son medios que tiene una presencia social que va más allá de las cifras de penetración o de inversión. Su capacidad educativa, de transmisión de valores y de creación de modas y tendencias es más elevada que la de otros medios (Medrano, Cortés y Palacios, 2007).

La estrategia publicitaria debería aprovecharse de esta circunstancia. Como único elemento negativo los medios convencionales deben contrarrestar un problema fundamental desde el punto de vista publicitario: la saturación. La propia Asociación Española de anunciantes demandó en 2009 que se redujera el tiempo de publicidad en televisión (Público, 2009). En noviembre de 2011 el Tribunal de Justicia de la Unión Europea condenó a España por permitir que la publicidad televisiva excediera los 12 minutos permitidos por ley.

\section{Gráfico 2. Evolución de la inversión en medios (convencionales / no convencionales)}

\begin{tabular}{|c|c|c|c|c|c|c|c|c|}
\hline 51,5 & 51 & 50,4 & 52,4 & 55,7 & 54,6 & 54,4 & 57,4 & 59,3 \\
\hline 48,5 & 49 & 49,6 & 47,6 & 44,3 & 45,4 & 45,6 & 42,6 & 40,7 \\
\hline 2005 & 2006 & 2007 & 2008 & 2009 & 2010 & 2011 & 2012 & 2013 \\
\hline
\end{tabular}

Fuente: Infoadex, 2014.

Nos encontramos, por tanto en un momento en el que la estrategia de medios resulta especialmente compleja y donde parece obligatoria la convivencia entre lo convencional y lo no convencional. Es necesario entender que el principal cambio se encuentra en el hecho de que los públicos se enfrentan a los medios con un actitud diferente, con una mayor carga crítica y con un alto nivel de independencia. Los datos pueden maquillar una realidad que queda constatada cuando la práctica de consumo se hace efectiva. Debemos entender que la transición de "públicos" a "consumidores" lleva aparejadas muchas modificaciones en el diálogo entre las marcas y los receptores.

\subsection{Los públicos del proceso publicitario}

El último eje en el proceso publicitario es, sin embargo, el más determinante. Como hemos observado en el epígrafe anterior los públicos han puesto en marcha una serie de cambios en cuanto al consumo de medios y en lo relativo al consumo en general. Así, la actual crisis económica parece estar afectando profundamente al modelo de consumo en nuestro país. Los cambios en el comportamiento del consumidor habían comenzado a producirse antes del estallido de la crisis por lo que resulta difícil discernir si se trata de una evolución natural o un cambio coyuntural derivado de la respuesta a una situación económica adversa (PWC, 2010). Los datos apuntan a que, pase lo que pase y sea cual sea el origen de la variación, el consumidor no volverá a ser el mismo, incluso si se produce la tan esperada recuperación 
económica (OMG, 2009). Por todo ello podemos hablar de cambios estructurales en los hábitos de consumo (Deloitte, 2011). La cuestión es identificar esos cambios y cómo se plasman en el proceso estratégico.

Para entender cuál es la realidad actual del consumo en nuestro país, debemos establecer una serie de condicionantes previos tanto desde el punto de vista cuantitativo como cualitativo. En el momento actual España vive una situación compleja en lo referido al consumo. Dicha situación está influenciada, en primer lugar, por una serie de condicionantes fruto de la evolución natural del mercado:

- Condicionantes demográficos: el estancamiento de la población limita el crecimiento de la demanda, por lo que, de una forma simplificada, podemos afirmar que son necesarios más individuos para que se puede producir un aumento del mercado: "mientras la población se reduzca, su fuerza laboral y el poder del consumo nacional se contraerá proporcionalmente, debilitando el potencial crecimiento del PIB real del país" (Deloitte, 2011). De esta forma, en los países en desarrollo (incluido España), los mercados se encuentran en un situación de madurez que hace muy difícil conseguir un crecimiento significativo.

- Condicionantes económicos: independientemente de la crisis económica, el consumidor español ha demostrado una baja confianza en la evolución del país. Los índices de confianza del consumidor registran sus peores niveles desde que existe referencia de esos datos. Bien es cierto que es índice marca tendencia a la baja desde hace diez años (GFK, 2012). En España, la percepción del consumidor sobre la situación económica es peor que la media europea, pesimismo que aumenta año tras año (Deloitte, 2011). A todo ello debemos sumar una percepción de que el desempleo y a situación económica son los principales problemas del país y se subraya la total desconfianza en la clase política para liderar y tomar medidas adecuadas de salida de la crisis (CIS, 2013).

- Condicionantes personales: todos los datos nos indican que el consumidor ha modificado sus valores personales de tal forma que se hacen más presentes los valores conservadores y de protección, la necesidad de relaciones estables, la valoración del entorno más próximo... (GFK, 2012). La importancia dada a la ecología y el medio ambiente supone la imposición de un modelo sostenible donde "los valores del consumidor en lo que se refiere a este aspecto permanecieron notablemente constantes durante la recesión y cada vez son más quienes se vuelven participantes activos" (Deloitte, 2011).

A partir de estos condicionantes generales, existe una serie de condicionantes específicos en el consumidor. Según el estudio elaborado en 2012 por Onmicon Media Group, gastan menos, pero compensan con mucha inteligencia la limitación del recurso. De esta forma, nace lo que se puede denominar smartshoper condicionado por una serie de factores que recogen lo indicado por el Instituto Nacional de Consumo en 2011 donde, además de los referidos cambios demográficos, económicos y personales, se hacia referencia a una serie de características del consumidor de este siglo:

- Escepticismo: ya en 2011 el 71\% de los consumidores creía que España estaba en recesión o a punto de entrar en ella (frente al $67 \%$ de la Unión Europea). Esto supone que el consumidor estaba muy alerta ante la posibilidad de que la crisis económica le afectara de forma muy directa.

- Deslealtad: 7 de cada 10 españoles está dispuesto a seguir comprando su marca favorita, pero no a cualquier precio. La importancia de la variable precio ha llevado a una mayor promiscuidad con las marcas, aunque muchos prefieren reducir la frecuencia de compra. 
- Orientación al valor: se puede hablar de una recuperación de los valores esenciales. Para el español las principales preocupaciones son la familia y la salud. Esto supone una oportunidad para el "renacimiento" de las marcas y productos tradicionales, que forman parte de los recuerdos familiares. Vida saludable y ecología son los siguientes de la lista.

Frente a estos elementos, que parecen ser consecuencia de la propia evolución del mercado, existen una serie de parámetros que sí ha variado notablemente como consecuencia de la crisis:

- Información. Se trata de un consumidor que invierte mucho más tiempo que antes en comprar (2 de cada 5 tiende a comparar precios en las páginas web, igualando la media europea). Esto supone un proceso de compra mucho más lento, espaciado y meditado. Frente al modelo anterior "una parte importante de las decisiones de compra se tomarán en el punto de venta. No tanto porque no se programen si no porque se espacian, se planean como actividades lúdicas" (INE, 2001).

- Confianza. El consumidor ha reducido notablemente su círculo de influenciadores en el proceso de compra. Es decir, antes se guiaba más por el efecto trendy de las marca, la sociedad... en cambio ahora parece haberse reducido el círculo de personas en las que confía como prescriptoras, recurriendo en un $42 \%$ a la recomendación de amigos más cercanos y familia.

- Compartir. 1 de cada 3 españoles comparte cosas con la familia y amigos: compartir, alquilar, tomar prestado, hacérselo uno mismo... Como ejemplo, el 22\% reconoce coger libros prestados en la biblioteca más que antes.

- Austeridad. Se evitan los lujos superfluos. El consumismo descontrolado está mal visto y 3 de cada 4 personas quieren comprar sólo las cosas que realmente necesitan o importan.

- Estilo de vida. Se vuelven a celebrar las fiestas en casas, a la comida casera (el 36\% de los consumidores cocinan ahora en casa, el $43 \%$ se lleva la comida al trabajo - frente al $36 \%$ de la media europea-). Como consecuencia se produce un aumento de la demanda de productos de "gran consumo".

Debemos hacer una especial referencia en lo relativo a los cambios de comportamiento del consumidor ya que debemos tener en cuenta lo indicado anteriormente: existen modificaciones coyunturales de respuesta a la crisis que conviven con cambios más estables y estructurales. Es cierto que el consumidor ha puesto en marcha una serie de fórmulas para hacer frente a la crisis. Así, según los datos de GFK (2012), se han establecido una serie de "recortes" en sus gastos, siendo el consumo de ocio el segmento más afectado. La única variación positiva se produjo, como hemos indicado, en alimentación, contradiciendo las estimaciones que indicaban que iba a ser la partida donde más se iba a reducir la inversión a comienzos de este siglo (INE, 2001).

Pero además de los recortes directos sobre los fondos disponibles, se han implementado una serie de fórmulas de ahorro con implicaciones muy profundas en el modelo de consumo español ya que suponen nuevos hábitos. El primer cambio se centra en la preferencia hacia productos más económicos y, como consecuencia, una mayor infidelidad hacia las marcas a lo que se añade una búsqueda activa de promociones. Se trata de cambios sutiles que, sin embargo, suponen un gran giro en la relación de fuerzas del mercado.

Las investigaciones anteriores confirman que, en este sentido, los cambios en el consumo guardan vinculación directa con una evolución del paradigma de relación de los públicos con los medios de comunicación, donde se inserta el concepto de prosumidor (Tofler, 1980) y la participación mediática de los consumidores supone que el proceso empresarial comercial y comunicativo deba adaptarse a una nueva realidad (Sánchez y Contreras, 2012). 


\section{Conclusiones}

Después del breve repaso realizado a un asunto tan amplio y complejo como es la aplicación de estrategias publicitarias, podemos retomar las hipótesis iniciales de la investigación para poder refutar o desechar cada una de ellas. Siguiendo el orden en que fueron planteadas y que coincide con su análisis en el trabajo podemos establecer lo siguiente:

- El proceso de planificación estratégica publicitaria no ha variado su estructura. De hecho, es el proceso de subordinación de estrategias el que parece estar condicionando la evolución del sector publicitario en nuestro país, con una preferencia de las estrategias promocionales sustentada en la presión de las estrategias de marketing relativas al precio.

- En lo referido a los sujetos del proceso publicitario, y especialmente en lo relativo a los medios de comunicación y los públicos, nos enfrentamos a cambios estructurales que van más allá de la coyuntura económica actual. Podemos empezar a hablar de una nueva relación de fuerzas en el mercado que condicionan el proceso comercial.

- Las estrategias publicitarias clásicas siguen siendo válidas produciéndose los avances en lo relativo a la ejecución de las mismas mediante el empleo de nuevos ejes o argumentos de venta.

- La lectura de los datos recogidos en las páginas anteriores coinciden en reforzar lo apuntado por la Nueva Teoría Estratégica en 2001, de tal forma que la recuperación del ser humano para la comunicación publicitaria es vital para su correcto funcionamiento, al igual que es fundamental el establecimiento de relaciones de "igual a igual" entre la marca y los públicos siendo la primera más dialogante, negociadora, cooperativa y consensual.

- Los relativo a la Nueva Teoría Estratégica queda demostrado en el breve trabajo de campo aplicado a cada uno de los sujetos publicitarios:

- Los anunciantes habían avanzado mucho en la gestión de la marca (brand management) si bien pronto han sido conscientes de la necesidad de que esa gestión pase por una adaptación a las necesidades reales del consumidor ya que esta es la única vía para conseguir el tan necesario "valor de marca".

- Las agencias de publicidad se mantienen en el contexto incierto en que llevan sobreviviendo décadas y aportan campañas y propuestas en las que el consumidor cobra verdadero protagonismo. Se trata de estrategias clásicas desarrolladas en torno a los insights o valores del consumidor.

- Los medios de comunicación son las principales víctimas de ese protagonismo del consumidor y a pesar de que la relación de fuerzas del mercado se mantiene, estos deben convivir con nuevas tendencias de consumo (multipantalla, online...) en los que la pauta viene marcada ya no por la audiencia si no por los seres humanos independientes.

- El público ha tomado conciencia clara de su papel de consumidor, adoptando un papel activo y convirtiéndose en un smartshoper que aplica estrategias de consumo muy claras y también en un prosumidor que genera contenido sobre la marca.

- Las estrategias publicitarias tienen asegurada su pervivencia mediante la aplicación de los preceptos recogidos en la Nueva Teoría Estratégica. La consideración de los receptores del mensaje publicitario como seres críticos e independientes permitirá encontrar vías de diálogo que doten de sentido a la comunicación comercial. Se trata de un proceso complejo y lento pero que ha empezado ya a dar algunos frutos. 
Pero además de la respuesta a las hipótesis de partida, el desarrollo de la investigación sobre estrategia publicitaria en general y en lo relativo a este caso en particular ha dado lugar a otras conclusiones adicionales:

- La evolución del concepto de estrategia ha ido paralela a la evolución del marketing como disciplina e incluso de la propia publicidad. Como resultado, la estrategia publicitaria cuenta con vestigios de las fases anteriores, especialmente en la adopción de fórmulas militares para su diseño o en las consideraciones economicista o corporativas, sin perder de vista los sesgos culturales y, por supuesto, la importante valoración de los intangibles.

- La crisis económica que asfixia el sector publicitario ha sacado a la luz procesos que parecían ir evolucionando de manera sigilosa. La reducción de la inversión en los medios convencionales es algo casi natural y supone la aceptación de la irrupción de un gran abanico de posibilidades comunicativas vinculadas con lo digital.

- Los anunciantes parecen confirmar su desconfianza en la publicidad mediante la desinversión, sin embargo son conscientes de la necesidad de establecer un diálogo con sus clientes que les permita recuperar el valor de sus marcas. De esta forma la responsabilidad parece estar en los responsables de diseñar las estrategias de comunicación (sean en la propia empresa o en agencias de publicidad) que deben buscar las vías adecuadas para establecer ese diálogo y plantear el futuro viable de la actividad publicitaria.

- Las agencias de publicidad han hecho pequeñas aportaciones para la búsqueda de soluciones publicitarias. De esta forma el mayor protagonismo del cliente, la asunción de la realidad social mediante el storytelling o la creación de mensajes sustentados en valores tradicionales parecen haber funcionado adecuadamente.

- Las incógnitas en cuanto a la evolución de los medios de comunicación resultan demasiado abrumadoras para ser abordadas en una investigación de carácter general, presentándose como necesaria una línea de trabajo específica que pueda prever los cambios futuros.

- El papel protagonista del receptor / público / consumidor es incuestionable y los cambios producidos hasta ahora parecen ser de no retorno. El prosumidor activo y crítico es el que puede haber provocado el caso existente en cuanto a la implementación de modelos estratégicos estables. Las estrategias publicitarias clásicas perviven, pero siempre atendiendo a los gustos, necesidades, intereses y valores (insights) que marque este nuevo consumidor.

\section{Referencias bibliográficas}

[1] AAKER, D. y MAHESWARAN, P. (1997). The effect of cultural orientation on persuasion. Journal of Consumer Research, 24, diciembre, 315-328.

[2] ANSOFF, H.; DECLERCK, R. y HAYES, R.L. (1976). From strategic planning to strategic management. Nueva York (USA): John Wiley \& Sons.

[3] BENAVIDES DELGADO, J. (1993). La crisis de la publicidad. Madrid: Edipo.

[4] CIS (2013). Barómetro del CIS.

[5] DELOITTE (2011). El consumidor 2020.

[6] FERNÁNDEZ, E.; ALAMEDA, D. y MARTíN, I. (2011). Las estrategias publicitarias de las marcas en contexto de crisis. Adcomunica, 1, 119-138.

[7] GARCIA UCEDA, M. (2005). Las claves de la publicidad. Madrid: ESIC. 
[8] GARCÍA, M.T. (2008). Políticas públicas y televisión digital. El caso de la TDT en España y el Reino Unido. Madrid: CSIC.

[9] GFK (2012). Cambios en los hábitos de consumo en periodo de crisis.

[10] GRUPO CONSULTORES (2012). AgencyScope. La relación agencia-anunciante en España a través del estudio de imagen de agencias desde 1978 a 2008.

[1 1] INE (2001). Tendencias del consumo y el consumidor en el siglo XXI.

[12] INFOADEX (2009). Resumen de la inversión publicitaria en España, 2009. Disponible en: http://www.infoadex.es/RESUMEN\%202009.pdf

[13] ITAMI, H. (1987). Mobilizing invisible assets. NY (USA): Harvard University Press.

[14] LEGERÉN LAGO, B. y GARCÍA-MIRÓN, Silvia (2012). Cambios y tendencias de la publicidad a nivel mediático. Más con menos. Icono 14, vol. 10(3). Disponible en: http://dx.doi.org/10.7195/ri14.v10i3.185

[15] LÓPEZ DE AGUILETA, C. y TORRES-ROMAY, E. (2007). Estrategias publicitarias alternativas dirigidas a los jóvenes en España: análisis de casos en revistas. Revista ALAIC, 6. Disponible en: http://www.eca.usp.br/asocia/alaic/revista/n6.htm

[16] MARTA, C. y GABELAS, J.A. (2008). La televisión como epicentro de la convergencia entre pantallas. Enlace. Revista venezolana de información, tecnología y conocimiento, vol. 1(5), 11-23.

[17] MEDRANO, C.; CORTÉS, M.D. y PALACIOS, S. (2007). La televisión y el desarrollo de valores. Revista de Educación, 342, 307-328.

[18] MORGERSTERN, O. y NEUMAN, J.V. (1994). Theory of Games and Economic Behavior. NJ (USA): Princeton University Press.

[19] NARAYADOU, M. (2006). Publicidad y crecimiento económico. Tesis doctoral en Economía. Universidad Paris 9.

[20] NTE (2014). Cátedra itinerante de la Nueva Teoría Estratégica. Disponible en: http://nuevateoriaestrategica.com/cinte/

[21] OMG (2009). Evolución del consumidor ante la recesión económica. Omnicon Media Group.

[22] OSIO ESQUIVEL, S. (2008). Breve historia de la publicidad. Encuadre. Revista de enseñanza del diseño, vol. 2, abril.

[23] PÉREZ RUIZ, A. (2001). Crisis en la estructura de la publicidad española. Área abierta, 1, noviembre.

[24] PÉREZ, R. A. (1996). Estrategia publicitaria y de las Relaciones Públicas. Madrid: Universidad Complutense de Madrid, Facultad de Ciencias Sociales y de la Comunicación.

[25] PÉREZ, R. A. y MASSONI, S. (2009). Hacia una teoría general de la estrategia. El cambio de paradigma en el comportamiento humano, la sociedad y las instituciones. Madrid: Ariel.

[26] PÉREZ, R.A. (2001). Estrategias de comunicación. Barcelona: Ariel.

[27] PORTER, M.E. (1980). Competitive Strategy. Nueva York (USA): Free Press.

[28] PRAHALAD, C.K. y HAMEL, G. (1994). Competing for the future. Harvard Business Review.

[29] PÚBLICO (2009). Las marcas piden menos publicidad en televisión. Público, 21 de octubre.

[30] PWC (2010). ¿Evolución o Revolución? El consumidor ante la crisis. Price Waterhousa Coopers. 
[31] SÁNCHEZ, J. Y CONTRERAS, P. (2012). De cara al prosumidor: producción y consumo empoderando a la ciudadanía 3.0. Icono 14, vol. 10(3), 66-84. Disponible en: http://dx.doi.org/10.7195/ri14.v10i3.210

[32] TOFLER, A. (1980). La tercera ola. Bogotá (Colombia): Plaza \& Janés.

[33] TORRES, E. y MUÑÓZ, C. (2006). Estrategias de posicionamiento basadas en la cultura del consumidor: un análisis de la publicidad en televisión. Estudios Gerenciales, 100, juliosepiembre, 71-81.

[34] TORRES-ROMAY, E. (2005). Estrategias publicitarias en procesos de crisis. Aplicación de un modelo de análisis teórico en casos reales. Fisec_Estrategias, 2.

[35] TORRES-ROMAY, E. (2007). La evolución histórica de la estrategia como base. La situación actual de las estrategias publicitarias. El largo camino hacia el brand management. Revista Fisec_Estrategias, 8. Disponible

en: http://www.cienciared.com.ar/usr/9/258/fisec8m5_pp3_26.pdf

[36] TORRES-ROMAY, E. (2009). La crisis del sector publicitario en España. En CAMPOS-FREIRE, F. (coord.), El cambio mediático (pp. 107-128). Sevilla: Comunicación Social.

[37] TORRES-ROMAY, E. (2010a). La influencia de la crisis en las estrategias comunicativas de las empresas españolas. Revista Fisec_Estrategias, 14. Disponible en: http://www.cienciared.com.ar/ra/usr/9/258/fisec_estrategias_n14m1pp3_30.pdf

[38] TORRES-ROMAY, E. (2010b). Publicidad y crisis: estrategias eficaces para el desarrollo de campañas publicitarias en contextos de crisis. En PERLADO, M. y JIMENEZ, C. (coord.), Escenario actual de la investigación en comunicación: objetivos, métodos y desafíos (pp. 360-378). Madrid: Edipo.

[39] TORRES-ROMAY, E. (2010c). Publicidad y precio. Estrategias promocionales como respuesta a la crisis. Revista de Estudios de Comunicación, vol. 11 (26), 183-197.

[40] TORRES-ROMAY, E. (2011). Advertcrisis: nuevas estrategias publicitarias para hacer frente a la crisis. En SIERRA SÁNCHEZ, J. y LIBERAL ORMAECHEA, S. (coord.), Reflexiones científicas sobre cine, publicidad y género desde la perspectiva audiovisual (pp. 291-308). Madrid: Fragua.

[41] TORRES-ROMAY, E. (2012). Tendencias actuales en la producción publicitaria. La evolución de las estrategias publicitarias y su reflejo en os procesos de producción. En CUESTA, U. (coord.), Planificación estratégica y creatividad (pp. 167-188). Madrid: ESIC. 